\title{
Ectopic Calcification in Uremia: Where Do We Stand?
}

\author{
Mario Cozzolino ${ }^{a}$ Paola Ciceri ${ }^{b}$ \\ ${ }^{a}$ Renal Division, Department of Health Sciences, ASST Santi Paolo e Carlo, University of Milan, Milan, Italy; \\ ${ }^{b}$ Department of Nephrology, Renal Research Laboratory, Dialysis and Renal Transplant, Fondazione Ca' Granda \\ IRCCS, Ospedale Maggiore Policlinico, Milan, Italy
}

Cardiovascular (CV) disease is one of the major causes of death in chronic kidney disease (CKD) patients [1]. The high CV risk is strongly associated to excess vascular calcification (VC), which is further observed in hemodialysis (HD) patients [2]. In addition, several studies suggest that oral calcium $(\mathrm{Ca})$ intake, particularly by $\mathrm{Ca}$-containing phosphate $(\mathrm{P})$ binders, worsens $\mathrm{VC}$ among $\mathrm{HD}$ patients. However, there is no specific therapy to prevent progression or to facilitate regression of VC, beyond careful attention to $\mathrm{Ca}$ and $\mathrm{P}$ balance, even if the strategy to use Ca-free $\mathrm{P}$ binders, especially in $\mathrm{HD}$ patients with extra-skeletal calcifications and uremic tumoral calcinosis (UTC), is strongly suggested.

Ca-P deposition, mostly in the form of hydroxyapatite, is the hallmark of VC and can occur in the arteries, cardiac valves, and myocardium. Ca-P deposits are present in distinct arterial layers and associate with specific pathologies [3]. In fact, medial calcification is an active process detectable in the course of ageing, renal insufficiency, and diabetes. It is often associated with mineral bone disorders, inflammatory status, and phenotypic switch of vascular cells. Compared to calcification of the medial layer, current evidences point to a different sequence of events driving calcification in atherosclerotic plaques. Intimal calcifications are secondary to inflammation, still dependent on vascular smooth muscle cells (VSMC) and macrophages infiltration into the early atheromatous plaques. Both intimal and medial arterial calcifications

karger@karger.com

(c) 2020 S. Karger AG, Basel

www.karger.com/bpu

Karger! occur in CKD population, with medial lesions that appear to be prevalent [2].

Two different mechanisms are proposed to explain the relationship between $\mathrm{Ca}$ and $\mathrm{P}$ disorders and VC: (1) the passive mechanism with Ca-P deposition in the vessels and (2) the active mechanism, through the induction of bone-related genes in VSMCs, that acquire bone-forming (osteoblast-like) cell phenotype [4]. Strong evidence suggests that $\mathrm{VC}$ is a highly regulated process, with both promoting and inhibiting processes involved [2]. Clearly, in vitro, cells derived from the tunica media undergo osteochondrogenic differentiation and matrix mineralization [5], suggesting that cell-mediated processes tightly control procalcific and anticalcific mediators in the vessels. The balance between inductors and inhibitors of calcification is fundamental to avoid ectopic calcification and maintain the normal artery physiology, even if the precise role of each inhibitor and inducer still needs further investigation.

An important role in the calcification process development is played by the uremic serum milieu with uremic toxins and phosphate as key regulators of $\mathrm{VC}$ in $\mathrm{HD}$. It has been demonstrated that serum from uremic patients either induces or accelerates Ca deposition in vitro due to the presence of a complex miscellaneous of uremic factors. Recently, it has been shown that there is a significant positive correlation between the degree of $\mathrm{VC}$ in vivo and the serum calcific potential, in high-Pi condition, in vitro 
through a higher potential to induce VSMCs osteoblastic trans-differentiation, apoptosis, and necrosis [6].

One of the main debated issues regarding VC is the possibility to block and eventually reverse already established VC in order to reduce CV disease morbidity and mortality in HD patients. Recently, in vitro studies on the effect of iron on VSMCs calcification have been published [7], accounting for an effect of iron in preventing highPi-induced VC. Interestingly, iron added when the calcification is already begun has the ability of totally stop the high-Pi-induced time-dependent exacerbation of $\mathrm{Ca}$ deposition.

One more interesting evidence is that CKD patients suffer from subclinical vitamin $\mathrm{K}$ deficiency [8], suggesting that this represents a population at risk for any biological consequences of poor vitamin $\mathrm{K}$ status. This deficiency might be caused by exhaustion of vitamin $\mathrm{K}$ due to its high requirement by vitamin $\mathrm{K}$-dependent proteins to inhibit VC. Considering the fact that vitamin K is essential for the activation of matrix Gla protein, an in situ inhibitor of tissue calcification, in HD patients functional vitamin $\mathrm{K}$ deficiency may contribute to the high VC burden.

In their paper, Guibao et al. [9] describe a retrospective analysis of $13 \mathrm{HD}$ patients affected by UTC, one of the more dramatic consequences of VC in CKD. Clearly, parathyroidectomy was able to reduce UTC in those HD patients affected by severe Secondary Hyperparathyroidism (SHPT), together with Ca-free P binder treatment and sodium thiosulphate. Interestingly, each patient with UTC receiving either sevelamer carbonate or lanthanum carbonate and sodium thiosulphate shows a significant improvement of ectopic calcification lesions.

Other factors, such as iron deficiency, vitamin $\mathrm{K}$ deficiency, and inflammations, should be considered in inducing UTC. The improved understanding into the pathogenesis of $\mathrm{VC}$ in uremia will give the opportunity to run new clinical studies to delay and treat this disease in HD population.

\section{Acknowledgment}

None.

\section{Disclosure Statement}

The authors have no conflicts of interest to declare.

\section{Funding Sources}

There are no funding sources to declare.

\section{Author Contributions}

Authors wrote the Editorial.

\section{References}

1 Okuno S, Ishimura E, Kitatani K, Fujino Y, Kohno K, Maeno Y, et al. Presence of abdominal aortic calcification is significantly associated with all-cause and cardiovascular mortality in maintenance hemodialysis patients. Am J Kidney Dis. 2007 Mar;49(3):417-25.

2 Vervloet M, Cozzolino M. Vascular calcification in chronic kidney disease: different bricks in the wall? Kidney Int. 2017 Apr;91(4): 808-17.

3 Cozzolino M, Gallieni M, Brancaccio D. Vascular calcification in uremic conditions: new insights into pathogenesis. Semin Nephrol. 2006 Jan;26(1):33-7.
4 Cianciolo G, Galassi A, Capelli I, Schillaci R, La Manna G, Cozzolino M. Klotho-FGF23, cardiovascular disease, and vascular calcification: black or White? Curr Vasc Pharmacol. 2018 Jan;16(2):143-56.

5 Ciceri P, Elli F, Cappelletti L, Tosi D, Savi F, Bulfamante G, et al. Osteonectin (SPARC) Expression in Vascular Calcification: In Vitro and Ex Vivo Studies. Calcif Tissue Int. 2016 Nov;99(5):472-80.

6 Ciceri P, Galassi A, Alfieri C, Messa P, Cozzolino M. Uremic Patients with Increased Vascular Calcification Score Have Serum with High Calcific Potential: Role of Vascular Smooth
Muscle Cell Osteoblastic Differentiation and Apoptosis. Blood Purif. 2019;48(2):142-9.

7 Ciceri P, Elli F, Braidotti P, Falleni M, Tosi D, Bulfamante $\mathrm{G}$, et al. Iron citrate reduces high phosphate-induced vascular calcification by inhibiting apoptosis. Atherosclerosis. 2016 Nov;254:93-101.

8 Cozzolino M, Mangano M, Galassi A, Ciceri P, Messa P, Nigwekar S. Vitamin K in Chronic Kidney Disease. Nutrients. 2019 Jan;11(1): E168.

9 Guibao et al. Treatment of Uremic Tumoral Calcinosis in Maintenance Hemodialysis Patients. Blood Purif. DOI: 10.1159/000506115. 